\title{
Numerical Prediction of Flow Patterns after Various Pipe
}

\section{Fittings}

\author{
Andreas Swienty, Raja Abou Ackl and Paul Uwe Thamsen \\ Department of Fluid System Dynamics, Berlin Institute of Technology, Berlin 10623, Germany
}

\begin{abstract}
An accurate prediction of flows using CFD depends on a large number of factors. In addition to discretizing the flow region, the correct definition of boundary or initial conditions and the choice of suitable numerical methods, the applied turbulence model influences the results of the flow simulation to a great extent. Therefore, a validation of the results with the experimental data is of great importance for a correct selection of a turbulence model. It is the scope of this paper to assess different turbulence models for the simulation of pipe flows. The calculation results of pipe flows through a combination of $90^{\circ}$ elbows and a $1 / 3$ segmental orifice are compared with experimental measurement results. This has the advantage that the suitability of the turbulence models for simulating both shear and swirl flows can be investigated. Thus, the k- $\omega$, k- $\varepsilon$ model and the Launder Reece Rodi Reynolds stress model are compared with each other and experimental results. Furthermore, this investigation is extended through including a much more $\mathrm{c}$ detached-eddy simulation. This model provides better prediction of the flow by resolving the large eddies and modeling the small ones. The experimental results originate from LDV measurements over the entire pipe cross-section. This measuring method provides velocity vectors over the measured surface.
\end{abstract}

Key words: CFD, turbulence models, $k-\varepsilon, k-\omega$, LRR, DES.

\begin{tabular}{|c|c|c|}
\hline \multicolumn{3}{|c|}{ Nomenclature } \\
\hline$c$ & [] & Turbulence parameter \\
\hline$D$ & {$[\mathrm{~m}]$} & Diameter of the pipe \\
\hline DES & & Detached eddy simulation \\
\hline$F$ & {$[N]$} & Force \\
\hline$g$ & {$\left[\mathrm{~m} \mathrm{~s}^{2}\right]$} & Acceleration of gravity \\
\hline$k$ & {$\left[\mathrm{~m}^{2} \mathrm{~s}^{-2}\right]$} & Turbulent kinetic energy \\
\hline LES & & Large eddy simulation \\
\hline LDV & & Laser doppler velocimetry \\
\hline LRR & & Launder Reece Rodi \\
\hline$p$ & {$[\mathrm{~Pa}]$} & Pressure \\
\hline PIV & & Particle image velocimetry \\
\hline $\begin{array}{l}R, r \\
\text { (U)RANS }\end{array}$ & {$[\mathrm{m}]$} & $\begin{array}{l}\text { Radius } \\
\text { (unsteady) Reynolds-averaged } \\
\text { Navier-Stokes }\end{array}$ \\
\hline$t$ & {$[\mathrm{~s}]$} & Time \\
\hline$u$ & {$\left[\mathrm{~m} \mathrm{~s}^{-1}\right]$} & Velocity \\
\hline$w$ & {$\left[\mathrm{~m}^{3} \mathrm{~s}^{-1}\right]$} & Flow rate \\
\hline
\end{tabular}

Greek Letters

$\begin{array}{lll}\varepsilon & {\left[\mathrm{m}^{2} \mathrm{~s}^{-3}\right]} & \text { turbulent energy dissipation rate } \\ \eta & {\left[\mathrm{m}^{2} \mathrm{~s}^{-1}\right]} & \text { dynamic viscosity }\end{array}$

Corresponding author: Andreas Swienty, M. Eng., research field: fluid system dynamics.

$\begin{array}{lll}\rho & {\left[\mathrm{kg} \mathrm{m}^{-3}\right]} & \text { Density of the fluid } \\ \sigma & {[]} & \text { Turbulence parameter } \\ \omega & {\left[\mathrm{s}^{-1}\right]} & \text { Specific rate of dissipation } \\ \text { Subscripts } & \text { and Superscripts } \\ \text { vol } & \text { Volumetric }\end{array}$

\section{Introduction}

In order to perform a reliable simulation of a flow problem with CFD, an understanding of the procedures and methods used is strongly needed. Apart from creating an incorrect mesh for the simulation model and a false indication of numerical methods, the choice of the turbulence model affects the outcome enormous. In addition, it is essential to validate the simulation results with measurements. The aim of this work is to investigate different turbulence models of the open source CFD program OpenFOAM for capturing pipe flows, which are disturbed by a segmental orifice and a combination of elbows based on measurement results. Elbow and segmental orifice fittings are the most common problems in pipes. 
Furthermore, these installations generate swirl and shear flows which require high demands on the turbulence model. The data used for comparison are measured on a test stand for investigating pipe flows by means of LDA. LDA measurements just as PIV measurements [1], are well suited for the study of highly vortical flows. Furthermore, these methods allow the capturing of large-scale as well as small-scale flow structures. Takamura [2] investigated the complex turbulent flow in a short elbow pipe under high Reynolds number conditions. In addition, Tanaka [3] carried out some URANS simulations of unsteady eddy motion in pipe flows at high Reynolds numbers conditions. Weissenbrunner [4] investigated the flow field after a 7\% segmental orifice by means of PIV measurements and transient detached eddy simulation as well as a stationary simulation with a RANS turbulence model. Vijiapurapu [5] considered the influence of different turbulence models for flows through rough pipes. With this work, an overview will be provided on how different turbulence models represent disturbed pipe flows.

\section{Experimental Setup}

In order to validate the simulation data, the described pipe fittings were examined on a test rig (Fig. 1). From a $4 \mathrm{~m}^{3}$ big tank water is pumped in a closed circuit by a centrifugal pump. The piping, with a diameter $D=53.6 \mathrm{~mm}$, is made primarily from PVC (Poly-Vinyl-Chloride). This allows a high flexibility of the test rig. The working fluid is tap water at $20^{\circ} \mathrm{C}$. A wide range of Reynolds numbers can be examined, since the pump generates a maximum flow rate of 42 $\mathrm{m}^{3} / \mathrm{h}$. In this study, a Reynolds number of 50,000 was used. In front of the measurement site a $100 \mathrm{D}$ long straight pipe ensures a fully developed flow. In addition, this is supported by the installation of a plurality of perforated plates and a tube bundle rectifier at the beginning of this pipe section. At the measuring section, a flow profile over the entire tube cross-section can be measured with Laser Doppler Velocimetry. The disturbance of the flow is directly induced by the installed fitting before the measuring section.

A $1 / 3$ segmental orifice with a thickness of $7 \mathrm{~mm}$ was investigated. In a segmental orifice a region of the tube cross section is closed by a segment. In this way a partially closed valve in pipelines can be imitated. The second examined geometry consists of two consecutive $90^{\circ}$ elbows (Fig. 2). The main function of an elbow is to redirect a pipe flow in a desired direction. At the interior side of an elbow the flow separates. This causes a complex flow field and vortices shedding downstream.

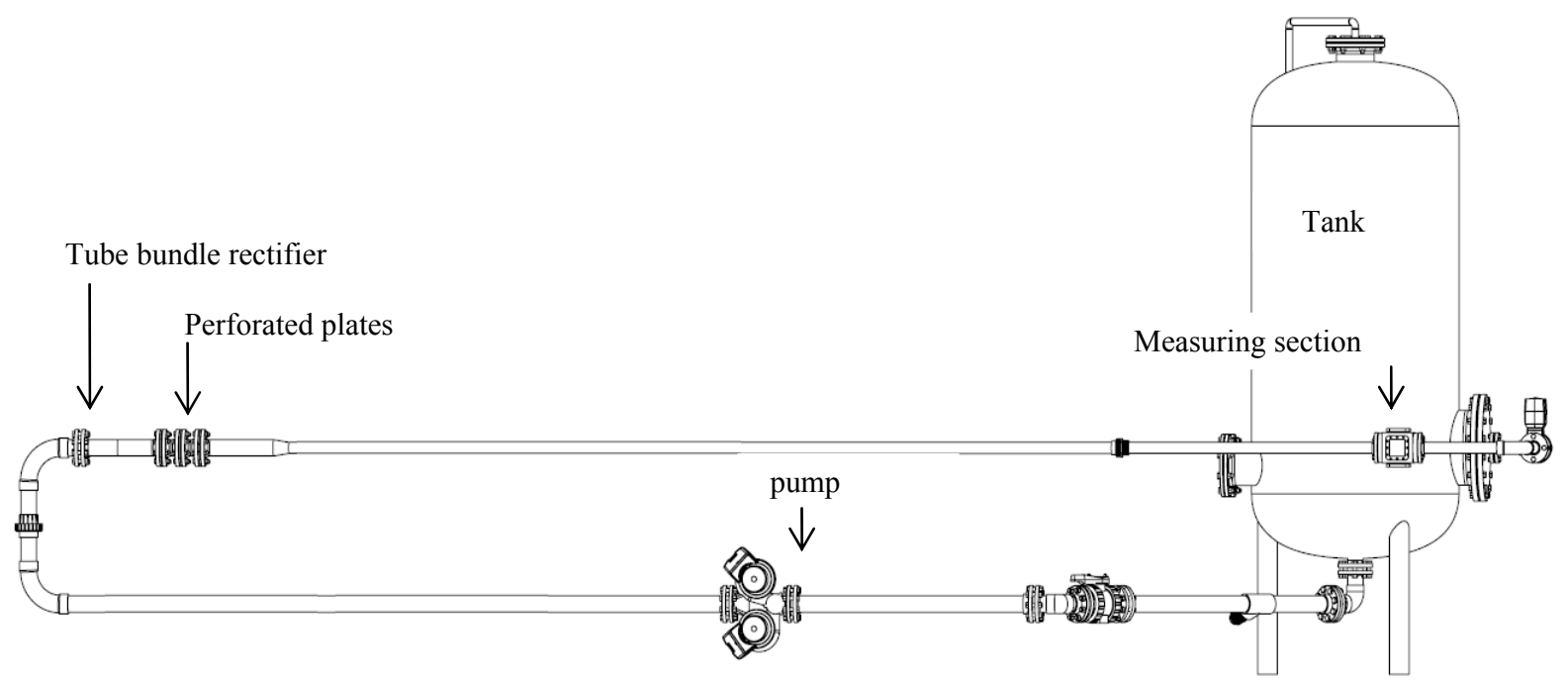

Fig. 1 Representation of the test stand for the investigation of flow profiles of pipe flows. 


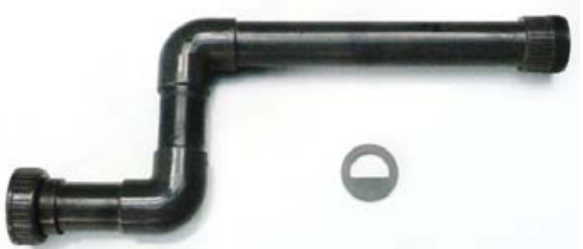

Fig. 2 The $1 / 3$ segmental orifice and the $90^{\circ}$ elbow combination made of PVC for the experiments on the test rig.

\section{Numerical Setup}

The swirling flow after the elbow and the shear flow after the segmental orifice are simulated with the same element size as well as boundary layers for the simulation grid and settings of the numerical method with the k-omega and the k-epsilon turbulence model and the LRR turbulence model (RANS model). In addition, these studies are extended by a computational-intensive detached eddy simulation. For this purpose the computational grid and the numerical methods were adapted to the requirements of the DES.

For an incompressible flow, the following continuity equation applies:

$$
\nabla \boldsymbol{u}=0
$$

Furthermore, the following can be written for the momentum equation:

$$
\rho \frac{\partial \boldsymbol{u}}{\partial t}=-\rho \boldsymbol{u} \nabla \boldsymbol{u}+\eta \nabla^{2} \boldsymbol{u}-\nabla p+\rho \boldsymbol{g}
$$

The $\mathrm{k}-\varepsilon$ turbulence model is the workhorse of practical engineering flow calculations to simulate mean flow characteristics for turbulent flow conditions [6]. It is a two equations model which gives a general description of turbulence by means of two transport equations. This approach consists of the two values $\mathrm{k}$ and $\varepsilon$. The equation $\mathrm{k}$, the turbulent kinetic energy, and $\varepsilon$, the rate of dissipation of the turbulent kinetic energy, have the following form:

and

$$
\frac{\partial \rho k}{\partial t}+\nabla(\rho k \boldsymbol{u})=\nabla\left(\frac{\eta_{t}}{\sigma_{k}} \nabla k\right)+\hat{P}-\rho \varepsilon
$$

$$
\begin{aligned}
\frac{\partial \rho \varepsilon}{\partial t}+\nabla(\rho \varepsilon \boldsymbol{u})= & \nabla\left(\frac{\eta_{t}}{\sigma_{\varepsilon}} \nabla \varepsilon\right)+C_{\varepsilon 1} \frac{\varepsilon}{k} \hat{P} \\
& -C_{\varepsilon 1} \rho \frac{\varepsilon^{2}}{k}
\end{aligned}
$$

In the $\mathrm{k}-\omega$ turbulence model, the turbulent kinetic energy is calculated after

$$
\begin{aligned}
\frac{\partial \rho k}{\partial t}+\nabla(\rho k \boldsymbol{u})= & \hat{P}-\beta^{*} \rho \omega \mathrm{k} \\
& +\nabla\left[\left(\eta+\frac{\eta_{t}}{\sigma_{k}}\right) \nabla k\right]
\end{aligned}
$$

and the specific rate of dissipation is calculated after

$$
\begin{aligned}
\frac{\partial \rho \omega}{\partial t}+\nabla(\rho \omega \boldsymbol{u}) & =\alpha \frac{\omega}{k} \hat{P}-\beta^{*} \rho \omega^{2} \\
& +\nabla\left[\left(\eta+\frac{\eta_{t}}{\sigma_{\omega}}\right) \nabla \omega\right]
\end{aligned}
$$

The equations contain some adjustable constants. The values of these constants originate from numerous iterations of data fitting for a wide range of turbulent flows. Through these two modeling approaches for the turbulence the required computational effort and resources are reduced. The Reynolds stress model [7, 8] involves calculations of the individual Reynolds stresses using differential transport equations. DES (detached-eddy simulation) is an example of a new kind of methods that seek to bridge the gap existing between the just introduced RANS approaches and LES (large-eddy simulation) concerning computational cost and predictive accuracy. In the regions next to walls RANS models can represent the flow very well at minimal computational cost, however, they exhibit significant deficiencies in massively-separated flows. On the contrary, LES delivers more accurate predictions due to the limitation of modeling empiricism to the smallest turbulent structures. However, the numerical cost for wall-bounded flows is enormous and increases strongly with Reynolds number.

In Fig. 3, the simulation model of the segmental orifice and the elbow combination is shown. For the segmental orifice, the data were evaluated at $7 \mathrm{D}$ and for the elbow combination $10 \mathrm{D}$ downstream of the disturbance.

For the stationary calculations with the k- $-\varepsilon, k-\omega$ and LRR turbulence model, a structured mesh with boundary layers was created with a total of 6.2 million elements. For the transient DES, this mesh was further refined, up to 13 million elements. Mostly, the 


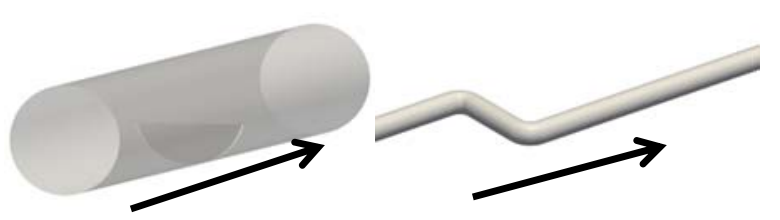

Fig. 3 Representation of the simulation geometry of the segmental orifice (l) and the elbow combination (r).
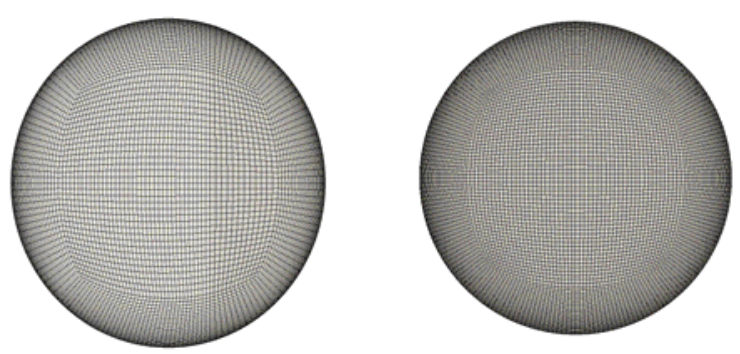

Fig. 4 Computational mesh for the segmental orifice (I) and the elbow combination (r).

element number was increased in the axial direction. A representation of the two meshes is shown in Fig. 4. The assessment of the independence of the results on the computational grid was checked by further calculations applying a general finer grid, here not presented.

At the inlet, a fully developed velocity profile was calculated with a periodic boundary condition. In this method the fully developed velocity profile is created by using a periodic boundary condition starting with a uniform velocity profile (Fig. 5). This was applied to the cases with the DES and RANS turbulence models. For the simulation, a kinematic viscosity of 1,004 $\times$ $10^{-6} \mathrm{~m}^{2} \mathrm{~s}^{-1}$ was selected for the property of the fluid.

\section{Results and Comparisons}

The results of the calculations of the $1 / 3$ segmental orifice are shown in the following Figs. 6-8. It is easy to see that the detached eddy simulation resolves more small structures than the simulations with the RANS turbulence model. In general, the simulations show a velocity profile, as it is expected downstream of a segmental orifice.

In addition, the data are evaluated along the axis of symmetry (Fig. 6). The velocity profiles along this axis are shown in Fig. 9.
Mapping the velocity values on the inlet patch

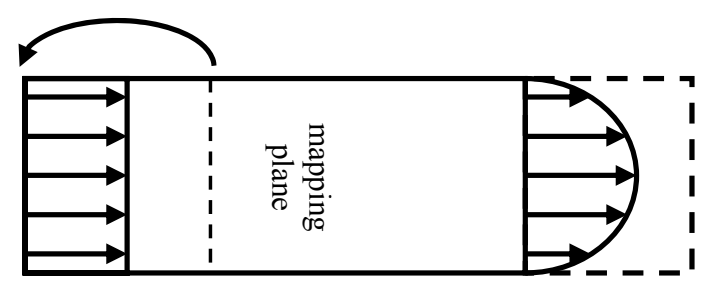
Uniform
velocity profile $\longrightarrow$ $\begin{aligned} & \text { Fully developed } \\ & \text { velocity profile }\end{aligned}$

Fig. 5 Schematic representation of the cycling mapped boundary condition.

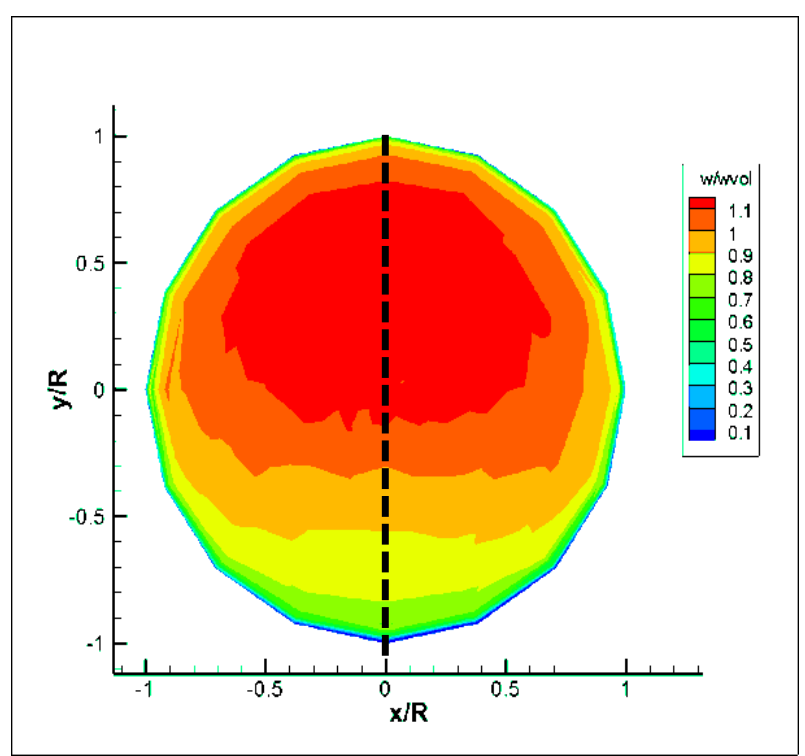

Fig. 6 Measurement result with LDV of the segmental orifice.

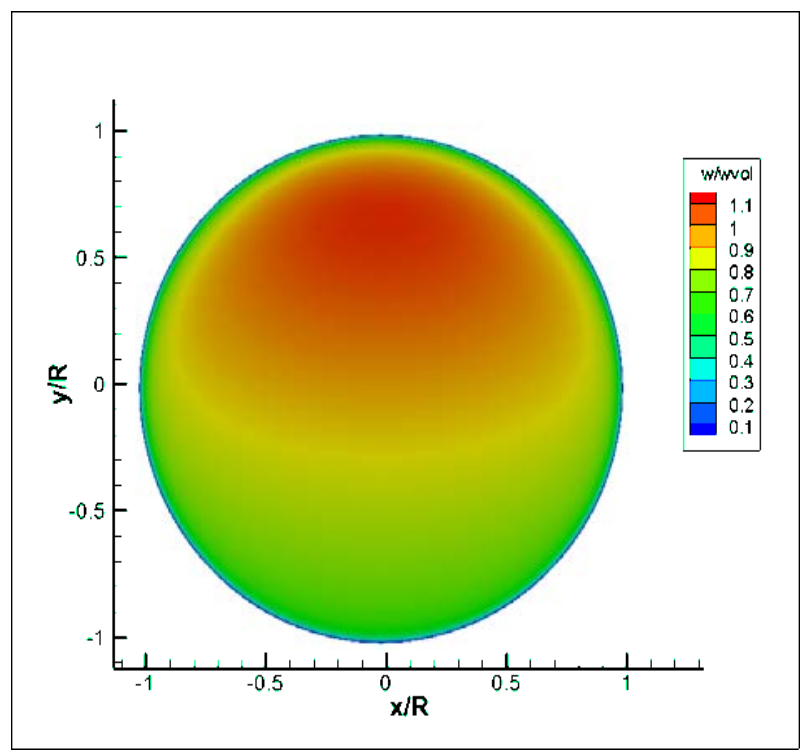

Fig. 7 Simulation result with the k- $\omega$ turbulence model of the segmental orifice. 


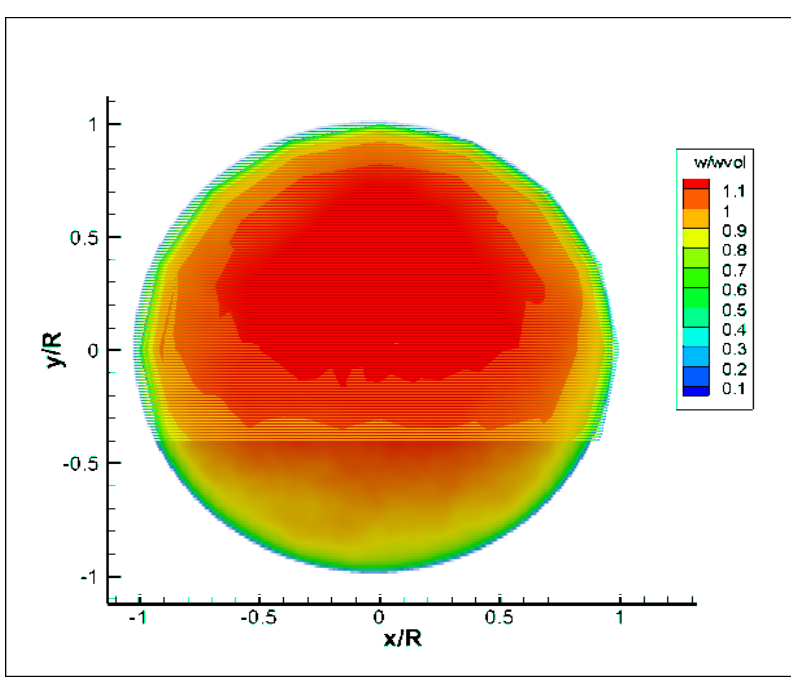

Fig. 8 Detached eddy simulation result of the segmental orifice.

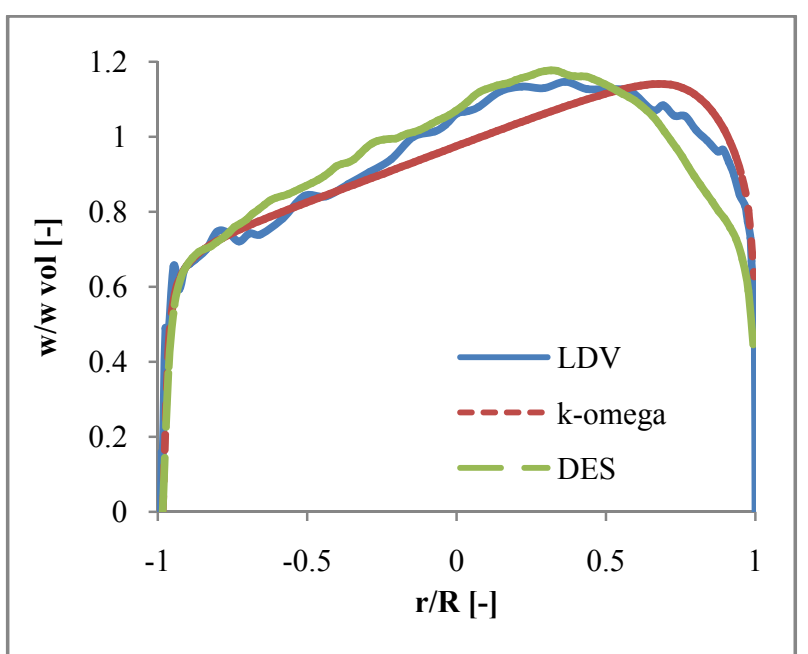

Fig. 9 Comparison of velocity profiles from different turbulence models of the segmental orifice.

The simulation results of the elbow combination are evaluated in the same manner. Figs. 10-14 show the velocity profiles.

In the elbow combination, all turbulence models were compared along one path (Fig. 10), too. The following chart (Fig. 15) shows the different velocity profiles.

\section{Summary and Conclusions}

The aim of this study was to investigate various turbulence models for the simulation of shear and swirl flows. In order to validate the simulation results measurements were performed on a $1 / 3$ segmental

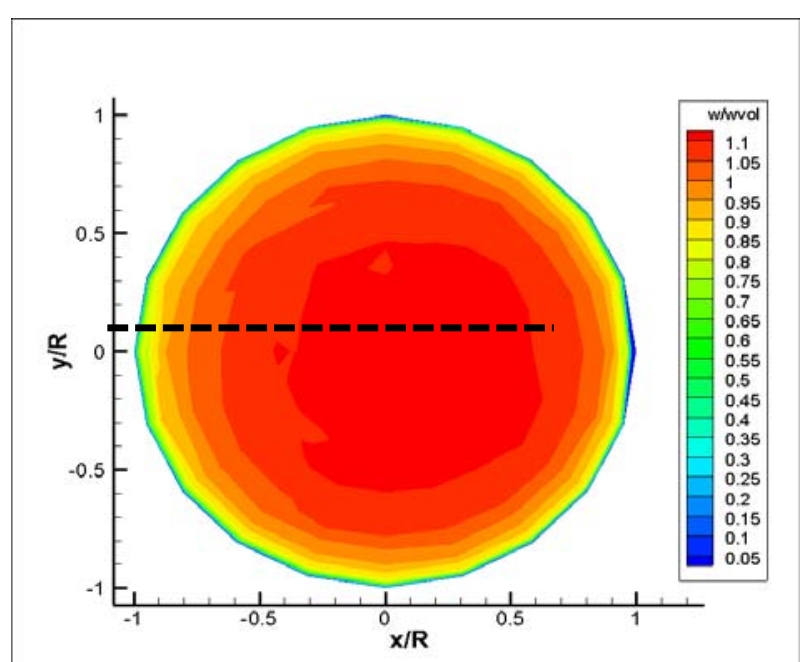

Fig. 10 Measurement result with LDV of the elbow combination.

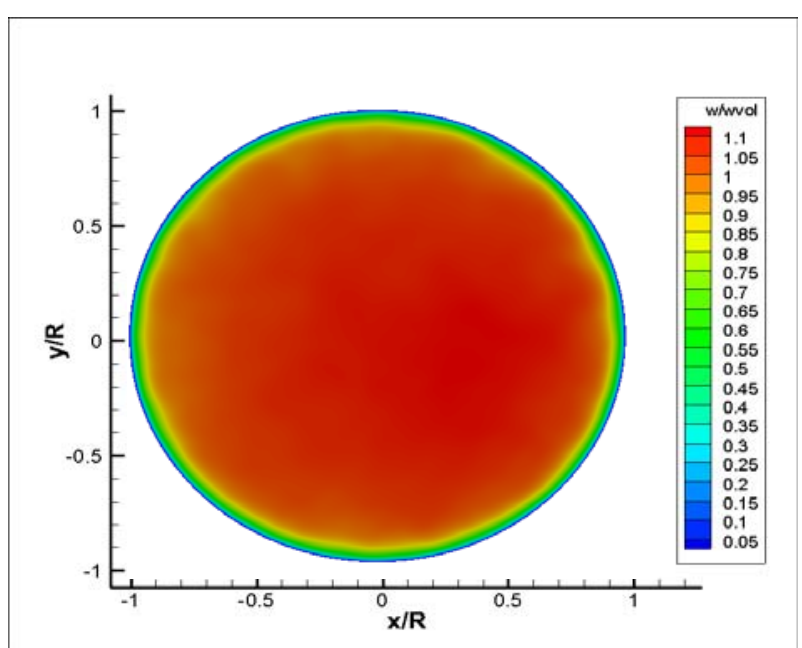

Fig. 11 Detached eddy simulation result of the elbow combination.

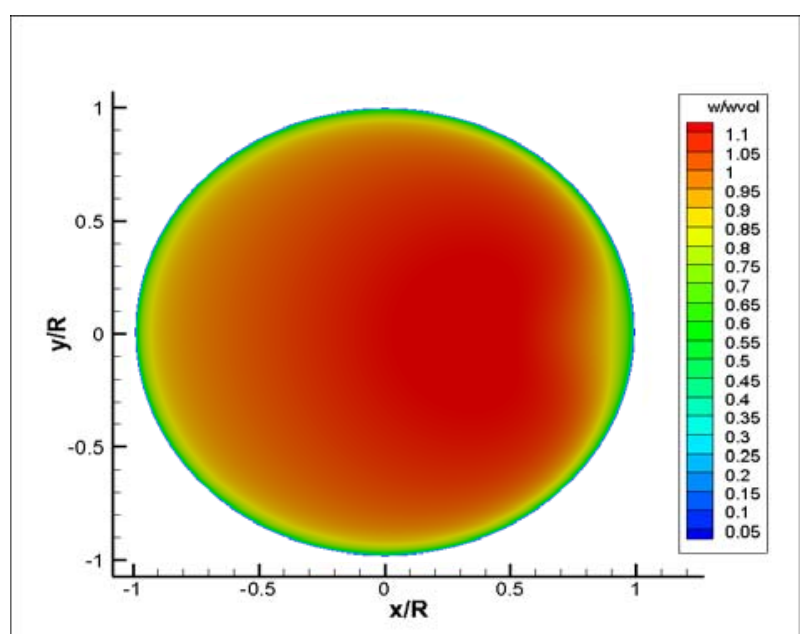

Fig. 12 Simulation result with the k- $\omega$ turbulence model of the elbow combination. 


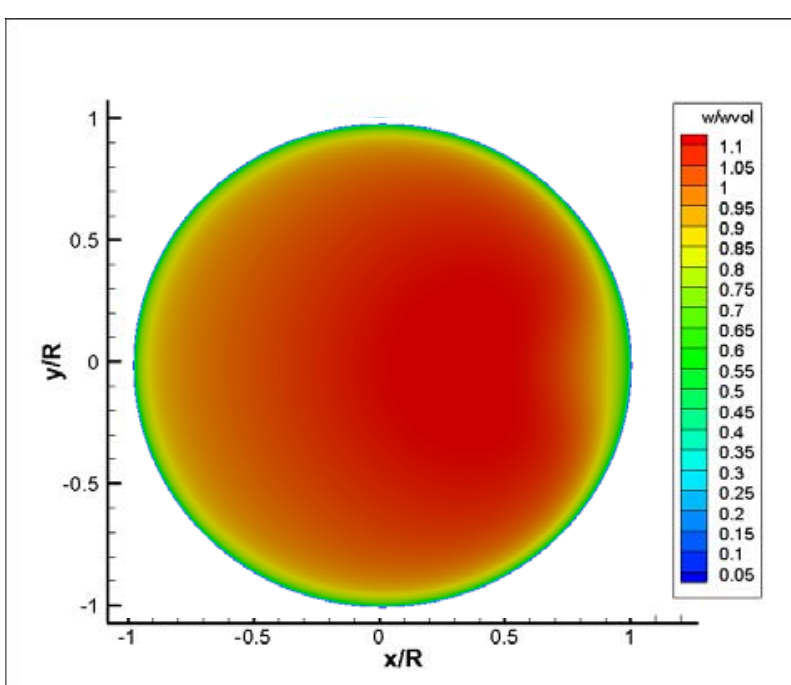

Fig. 13 Simulation result with the k- $\varepsilon$ turbulence model of the elbow combination.

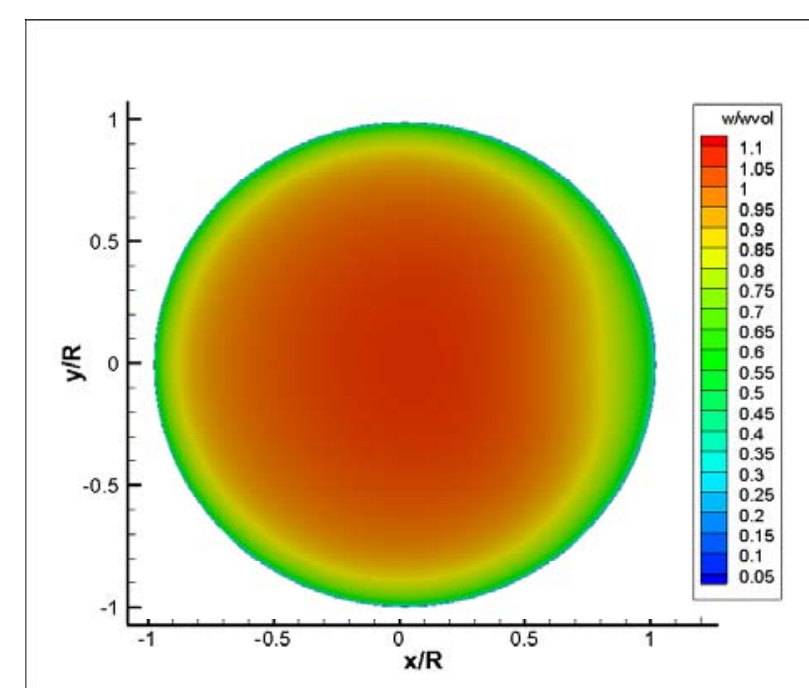

Fig. 14 Simulation result with the LLR turbulence model of the elbow combination.

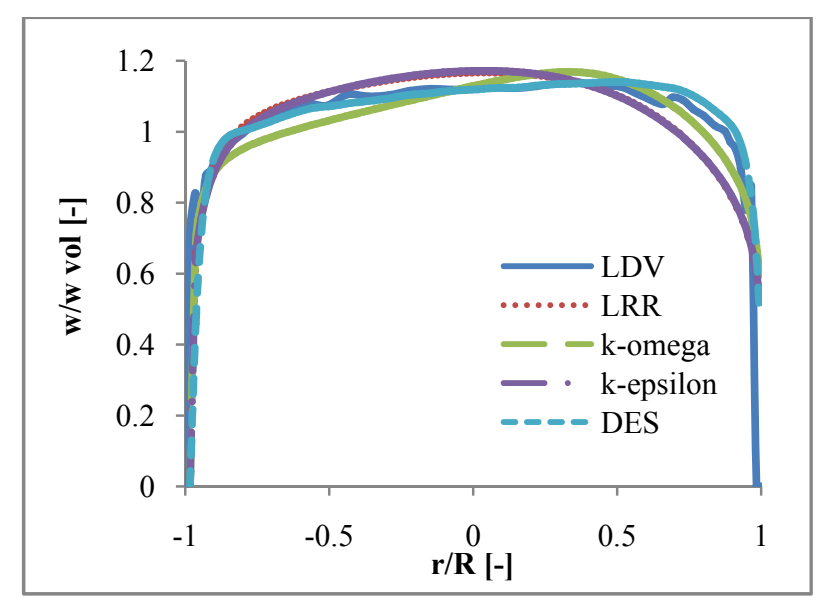

Fig. 15 Comparison of velocity profiles from different turbulence models of the elbow combination. orifice and a combination of $90^{\circ}$ elbows on a test rig for investigating pipe flows. The velocity profiles over the entire pipe cross section were measured by means of LDV. In a further step, the simulation models of the described disturbances were created. The k-omega, the k-epsilon, the LRR and the detached eddy simulation turbulence models were examined in this study for capturing the flow conditions.

The comparison of the simulation results showed that the computationally expensive detached eddy simulation resolves the flow very well both in the segmental orifice and in the elbow combination. Nevertheless, in the case with the segmental orifice this model shows some inaccuracy in the regions near to the wall. This can be lead back to the resolution of the mesh in this area.

Regarding the RANS turbulence models, the k- $\omega$ model showed a good correlation with the measurements. Concerning the elbow combination the LRR model performed just as poorly as the k- $\varepsilon$ model. However, the LRR model is suitable for resolving flows with high vortex intensity, therefore, it can be reasonably concluded that the exciting vortices after the elbow combination are not strong enough to use the benefits of this turbulence model.

\section{References}

[1] Sharp, K. V., and Adrian, R. J. 2001. "PIV Study of Small-Scale Flow Structure around a Rushton Turbine." A.I.Ch.E. Journal 47 (4): 766-78.

[2] Takamura, H., Ebara, S., Hashizume, H., Aizawa, K., and Yamano, H. 2012. "Flow Visualisation and Frequency Fluctuations of Complex Turbulebt Flow in a Short Elbow Piping under High Reynolds Number Condition." Journal of Fluids Engineering 134: 101201-1-101201-8.

[3] Tanaka, M., Ohshima, H., Yamano, H., Aizawa, K., and Fujisaki, T. 2010. "U-RANS Simulation of Unsteady Eddy Motion in Pipe Elbow at High Reynolds Number Condition." In Proc. 2010 International Congress on Advances in Nuclear Power Plants (CD-ROM), 1699-708.

[4] Weissenbrunner, A., Eichler, T., and Lederer, T. 2014. CFD Simulation of a Flow Disturber in a Pipe and Comparison with PIV-Measurement. Fachtagung: Lasermethoden in der Strömungsmesstechnik. 
[5] Vijiapurapu, S., and Cui, J. 2010. "Performance of Turbulence Models for Flows through Rough Pipes." Applied Mathematical Modelling 34: 1458-66.

[6] ANSYS, I. 2009. ANSYS Fluent 12 Users Guide.

[7] Launder, B. E., Reece, G. J., and Rodi, W. 1975. "Progress in the Development of a Reynolds-Stress
Turbulence Closure.” J. Fluid Mech. 68 (3): 537-66.

[8] Fu, S., Launder, B. E., and Leschziner, M. A. 1987. "Modeling Strongly Swirling Recirculating Jet Flow with Reynolds-Stress Transport Closures." Presented at the Sixth Symposium on Turbulent Shear Flows, Toulouse, France. 